\title{
On the Unique Solvability of a Volevič System of Linear Equations with General Singularity
}

\author{
Jose Ernie C. Lope, Jose Maria L. Escaner IV \\ and Carlene P. Arceo
}

\begin{abstract}
We consider a Volevič system of linear partial differential equations with general singularity, for which we establish existence and uniqueness theorems that are analogues of the Cauchy-Kowalevsky and Holmgren Theorems. Our results are generalizations of those of Elschner [Beiträge Anal. 12 (1978), 185 - 198], Lope [J. Math. Sci. Univ. Tokyo, 6 (1999), 527 - 538] and Tahara [J. Math. Soc. Japan 34 (1982), 279 - 288], which are in turn generalizations of the results of Baouendi and Goulaouic [Comm. Pure Appl. Math. 26 (1973), 455 - 475].
\end{abstract}

Keywords: Volevič system, singular partial differential equations

MSC 2000: Primary 35A10, secondary 35A20, 35G05

\section{Statement of the Problem and Main Results}

Let $\Omega$ be a bounded set in $\mathbb{R}^{n}$. Given any $r \in(0, R]$, we denote by $D(a, r)$ the disk $\left\{z \in \mathbb{C}^{n} ;\left|z_{i}-a_{i}\right|<r, i=1,2, \ldots, n\right\}$ and we define a neighborhood of $\Omega$ by $\Omega_{r}=\bigcup_{a \in \Omega} D(a, r)$. The set of all functions $u(z)$ holomorphic in $\Omega_{r}$ and continuous up to $\bar{\Omega}_{r}$ is a Banach space with norm given by

$$
\|u\|_{r}=\max _{z \in \bar{\Omega}_{r}}|u(z)|
$$

This space will be denoted by $\mathscr{A}\left(\Omega_{r}\right)$, or simply by $\mathscr{A}_{r}$.

Consider now the system of linear partial differential equations in independent variables $(t, z) \in \mathbb{R} \times \mathbb{C}^{n}$ given by

$$
\rho(t) D_{t} u+A\left(t, z, \mu(t) D_{z}\right) u=f(t, z) .
$$

Here, $D_{t}$ and $D_{z}$ denote the derivatives $\partial / \partial t$ and $\left(\partial / \partial z_{1}, \ldots, \partial / \partial z_{n}\right)$, respectively; $u=\left(u_{1}, \ldots, u_{m}\right)^{\top}$ is the unknown; $f=\left(f_{1}, \ldots, f_{m}\right)^{\top}$ belongs to the

All authors: Department of Mathematics, University of the Philippines, Diliman, 1101 Quezon City, Philippines; \{ernie, joma, cayen\}@math.upd.edu.ph

ISSN 0232-2064 / \$ 2.50 (c) Heldermann Verlag Berlin 
space $\mathscr{C}^{0}\left([0, T], \mathscr{A}_{R}\right)$, i.e., each $f_{i}$ is a continuous function on some interval $[0, T]$ that is valued in the space $\mathscr{A}_{R}$; and $A\left(t, z, \mu(t) D_{z}\right)=\left(A_{i j}\left(t, z, \mu(t) D_{z}\right)\right)$ is an $m \times m$ matrix of linear operators. All throughout this paper, we will assume the following:

(A1) The function $\rho(t)$ is continuous, positive on $(0, T]$, and satisfies

$$
\int_{0}^{T} \frac{1}{\rho(t)} d t=+\infty
$$

(A2) The function $\mu(t)$ is continuous, positive and nondecreasing on $(0, T]$, and satisfies the integrability condition

$$
\int_{0}^{T} \frac{\mu(t)}{\rho(t)} d t<+\infty
$$

(A3) The system (2) is a Volevič system of linear partial differential equations, i.e., there exists an $m$-tuple of nonnegative integers $\left(n_{1}, \ldots, n_{m}\right)$ such that for each $(i, j)$, the order of $A_{i j}$ is at most $n_{i}-n_{j}+1$. Thus the operator $A_{i j}$ has the form

$$
A_{i j}\left(t, z, \mu(t) D_{z}\right)=\sum_{|\alpha| \leq n_{i}-n_{j}+1} a_{i j, \alpha}(t, z)\left(\mu(t) D_{z}\right)^{\alpha} .
$$

We will further assume that each of the coefficients $a_{i j, \alpha}$ belongs in the space $\mathscr{C}^{0}\left([0, T], \mathscr{A}_{R}\right)$.

(A4) There is a constant $c>0$ such that

$$
\operatorname{Re} \alpha_{j}(z) \geq 2 c
$$

holds on $\bar{\Omega}_{R}$ for each eigenvalue $\alpha_{j}(z)$ of the matrix $A(0, z, 0)$.

Note that since the function $\mu(t)$ is nondecreasing and satisfies (3), we must have $\lim _{t \rightarrow 0} \mu(t)=0$. In [7], Tahara considered (2) with singularity $\rho(t)$ equal to $t^{\sigma}(\sigma \geq 1)$. In the specific case when $\rho(t)=t$, any function $\mu(t)$ satisfying assumption (A2) is referred to as a weight function by Tahara [8] (see also Lope [5]).

Under these assumptions, we can prove the following analogue of the wellknown Cauchy-Kowalevsky Theorem.

Theorem 1.1. Given any $r \in(0, R)$, there is a positive number $\epsilon \in(0, T)$ such that for any $f \in \mathscr{C}^{0}\left([0, T], \mathscr{A}_{R}\right)$, Equation (2) has a unique solution $u \in$ $\mathscr{C}^{0}\left([0, \epsilon], \mathscr{A}_{r}\right) \cap \mathscr{C}^{1}\left((0, \epsilon], \mathscr{A}_{r}\right)$ satisfying $\rho(t) D_{t} u \in \mathscr{C}^{0}\left([0, \epsilon], \mathscr{A}_{r}\right)$.

We can also formulate a dual version of Theorem 1.1 for analytic functionals. Using this dual version, we can follow the arguments of Baouendi and Goulaouic 
in [1] in order to prove an analogue of Holmgren's Theorem. Let $\Omega$ contain the origin in $\mathbb{R}^{n}$, let $A\left(t, x, \mu(t) D_{x}\right)$ be the restriction of the operator $A\left(t, z, \mu(t) D_{z}\right)$ on $[0, T] \times \Omega$, and let us consider the system

$$
\rho(t) D_{t} u+A\left(t, x, \mu(t) D_{x}\right) u=0
$$

in real independent variables $(t, x)$ and in the space $\mathscr{C}^{0}\left([0, T], \mathscr{D}^{\prime}(\Omega)\right)$. Here, $\mathscr{D}^{\prime}(\Omega)$ denotes the space of all distributions on $\Omega$. Then we have the following uniqueness result concerning distribution solutions of (5).

Theorem 1.2. Let $u \in \mathscr{C}^{0}\left([0, T], \mathscr{D}^{\prime}(\Omega)\right) \cap \mathscr{C}^{1}\left((0, T], \mathscr{D}^{\prime}(\Omega)\right)$ be any solution of (5). Then $u$ has to be zero in some neighborhood of $(0,0) \in[0, T] \times \mathbb{R}^{n}$.

Remark 1.3. The case when $\rho(t)=t^{\sigma}(\sigma \geq 1)$ and $\mu(t)=t^{\rho}(\rho>\sigma-1)$ has been considered by Elschner [2] and later by Tahara [7]. Our theorems also apply to higher-order scalar equations since such equations can be rewritten as a Volevič system of equations. In particular, the scalar equation

$$
\left(\rho(t) D_{t}\right)^{m} u+\sum_{\substack{j+|\alpha| \leq m \\ j<m}} a_{j \alpha}(t, x)\left(\mu(t) D_{z}\right)^{\alpha}\left(\rho(t) D_{t}\right)^{j} u=f(t, z)
$$

can be written in the form $(2)$ with $\left(n_{1}, \ldots, n_{m}\right)=(1, \ldots, m)$. Existence and uniqueness theorems have been proved for (6) by Baouendi and Goulaouic [1] for the case when $\rho(t)=t$ and $\mu(t)=t^{a}(a>0)$, and by Lope [5] for the case when $\rho(t)=t$ and for a general weight function $\mu(t)$. Hence, our theorems are generalizations of these previous results.

Remark 1.4. Towards the completion of this manuscript, the authors learned that Koike had established in [4] a local existence and uniqueness theorem in the ultradifferentiable class with respect to the space variable for a Volevič system of nonlinear equations. The linear version of his system is very similar to ours - it is our system with the $A_{i j}$ 's given instead by

$$
A_{i j}\left(t, z, \mu(t) D_{z}\right)=\sum_{\beta \in \Delta_{i j}(\lambda)} a_{i j, \beta}(t, z)\left(\mu(t) D_{z}\right)^{\beta} .
$$

In the equation above, $\lambda \geq 1$ is a fixed number chosen a priori for the whole system and $\Delta_{i j}(\lambda)$ is the set of multi-indices $\left\{\beta \in \mathbb{N}^{n} ; \lambda|\beta| \leq n_{i}-n_{j}+1\right\}$. He then proved existence and uniqueness under the following assumption on the functions $\mu(t)$ and $\rho(t)$ :

$$
\int_{0}^{T} \frac{\mu(t)^{\kappa}}{\rho(t)} d t<+\infty \quad \text { for some } \kappa \in\left(0, \frac{1}{\lambda}\right) .
$$

This integrability condition is stronger than the one stated in (3). One can easily verify that our theorem covers, for example, the case when $\rho(t)=t$ and 
$\mu(t)=(-\log t)^{-1}(\log (-\log t))^{-\nu}$ where $\nu>1$, but the result in [4] does not. So while Remark 2 in $\S 2$ of the paper says that the main theorem also applies to the class of analytic maps, the assumptions on the differential equations are strictly stronger than ours; we are therefore dealing with a more general system of linear equations.

\section{Preliminaries}

Our starting point is the system

$$
\rho(t) D_{t} u+A(t, z, 0) u=f(t, z)
$$

of ordinary differential equations. We can use standard techniques to see that this system is uniquely solvable, thus making it possible to define the inverse of the operator $\rho(t) D_{t}+A(t, z, 0)$. It is however important to go beyond establishing unique solvability; we have to establish estimates, similar to the ones obtained in [7], for the image of the inverse operator.

We begin by giving a precise statement of the existence and uniqueness result for (7). The proof is very similar to that in [7] and hence may be omitted.

Lemma 2.1. Let $\epsilon>0$ be sufficiently small and let $r \in(0, R]$. Then for any given function $f \in \mathscr{C}^{0}\left([0, \epsilon], \mathscr{A}_{r}\right)$, the system $(7)$ has a unique solution $u \in \mathscr{C}^{0}\left([0, \epsilon], \mathscr{A}_{r}\right) \cap \mathscr{C}^{1}\left((0, \epsilon], \mathscr{A}_{r}\right)$ satisfying $\rho(t) D_{t} u \in \mathscr{C}^{0}\left([0, \epsilon], \mathscr{A}_{r}\right)$.

Let $\mathscr{R}$ be the inverse operator of $\rho(t) D_{t}+A(t, z, 0)$, that is, given $f \in$ $\mathscr{C}^{0}\left([0, \epsilon], \mathscr{A}_{r}\right)$, we define $\mathscr{R}[f]$ to be the unique solution $u$ of $(7)$. We can view $\mathscr{R}$ as an $m \times m$ matrix of operators on the space $\mathscr{C}^{0}\left([0, \epsilon], \mathscr{A}_{r}\right)$. The $(i, j)$-th component of $\mathscr{R}$ will be denoted by $\mathscr{R}^{i j}$.

We will state an estimate for the image of $\mathscr{R}$ in terms of another operator which we will introduce shortly. Define $\kappa(t)$ on $(0, T)$ by

$$
\kappa(t)=-\int_{t}^{T} \frac{1}{\rho(\eta)} d \eta .
$$

Since $\rho$ is strictly positive, we see that $\kappa(t)$ strictly decreases from $-\infty$ to 0 on $(0, T]$. This implies the existence of its inverse function which increases from 0 to $T$ over the interval $(-\infty, 0]$.

Now, given a function $g(t)$ that is continuous on $[0, T]$, we define

$$
\mathscr{H}[g](t)=\int_{0}^{\infty} e^{-c s} g(\psi(t, s)) d s \quad(0 \leq t \leq T),
$$

where $c$ is the constant in (4) and $\psi(t, s)$ is equal to $\kappa^{-1}(\kappa(t)-s)$. The function $\psi(t, s)$ takes the place of $\phi_{\sigma}(t, s)$ in [7], and also possesses those properties 
of $\phi_{\sigma}(t, s)$ that are of interest to us. It is, for example, easy to check that $\psi\left(\psi\left(t, s_{1}\right), s_{2}\right)=\psi\left(t, s_{1}+s_{2}\right)$ holds, so that we have the following formula for the composition of $\mathscr{H}$ with itself $k$ times:

$$
\mathscr{H}^{k}[g](t)=\frac{1}{(k-1) !} \int_{0}^{\infty} s^{k-1} e^{-c s} g(\psi(t, s)) d s .
$$

Note further that $\psi(t, s) \leq t$ for any $s \in(0, \infty)$, and this maximum value is attained at $s=0$. Hence, if $g(t)$ is real-valued, nonnegative and increasing, then the estimate

$$
\mathscr{H}[g](t) \leq \frac{1}{c} g(t)
$$

holds for all $t \in[0, T]$. Finally, using a change of variable, we can also rewrite (8) as

$$
\mathscr{H}[g](t)=\int_{0}^{t} e^{-c[\kappa(t)-\kappa(s)]} g(s) \frac{d s}{\rho(s)} \quad(0 \leq t \leq T) .
$$

Since $\kappa$ is increasing, the exponential above is always less than 1 , and so we also have this estimate for any nonnegative real-valued function $g$ :

$$
\mathscr{H}[g](t) \leq \int_{0}^{t} g(s) \frac{d s}{\rho(s)} .
$$

We now state the following lemma which gives an estimate in terms of the operator $\mathscr{H}$ of the image of a function $g$ under $\mathscr{R}$. This is essentially Lemma 2 of Tahara [7] adapted to the operator $\rho(t) D_{t}+A(t, z, 0)$.

Lemma 2.2. Let $\epsilon>0$ be sufficiently small. There exists a constant $C>0$ such that the estimate

$$
\left\|\mathscr{R}^{i j}[g](t)\right\|_{r} \leq C \mathscr{H}^{p(i, j)}\left[\|g\|_{r}\right](t)
$$

holds for any $r \in(0, R]$, for any function $g \in \mathscr{C}^{0}\left([0, \epsilon], \mathscr{A}_{r}\right)$ and for all $t \in[0, \epsilon]$. Here, $p(i, j)=\max \left(1, n_{j}-n_{i}+1\right)$ and $\|g\|_{r}(t)=\sup _{0 \leq \tau \leq t}\|g(\tau, \cdot)\|_{r}$, where $\|\cdot\|_{r}$ is the norm defined in (1).

Proof. In view of (9), we can simply follow the arguments in the proof of Lemma 2 in [7] with $\phi_{\sigma}(t, s)$ replaced by $\psi(t, s)$.

Define the function $\varphi(t)$ on the interval $[0, T]$ by

$$
\varphi(t)=\int_{0}^{t} \frac{\mu(s)}{\rho(s)} d s .
$$

This definition makes sense in view of the integrability condition on $\mu(t)$. It is clear that $\varphi(t)$ is continuous and increasing on $[0, T]$ with $\varphi(0)=0$, and is differentiable in $(0, T)$.

The following corollary to Lemma 2.2 plays a very important role in the proof of Theorem 1.1. It gives an estimate of the action of the operator $\mathscr{R}$ when $g(t)$ satisfies a certain estimate in terms of $\mu$ and $\varphi$. 
Corollary 2.3. Suppose that for some $K \geq p(i, j)$ and $L \geq 0$, the function $g \in \mathscr{C}^{0}\left([0, \epsilon], \mathscr{A}_{r}\right)$ satisfies the estimate $\|g(t)\|_{r} \leq \mu(t)^{K} \varphi(t)^{L}$ for all $t \in[0, \epsilon]$. Then there is a constant $C>0$ such that for any integer $l=0,1, \ldots, p(i, j)$, we have

$$
\left\|\mathscr{R}^{i j}[g](t)\right\|_{r} \leq C\left(\frac{1}{c}\right)^{p(i, j)-l} \frac{\mu(t)^{K-l} \varphi(t)^{L+l}}{(L+1)_{l}} \quad(t \in[0, \epsilon]) .
$$

Here, we have employed the notation $(q)_{n}=q(q+1) \ldots(q+n)$.

Proof. We only have to use (10) and (11) in conjunction with Lemma 2.2. We further take note of the fact that $\varphi^{\prime}(t)$ is, by definition, equal to $\mu(t) / \rho(t)$.

Finally, we mention a lemma which is fundamental in estimating functions in a scale of Banach spaces.

Lemma 2.4 (Nagumo). Let $f(z)$ be holomorphic in $\Omega_{R}$. Suppose there are constants $K>0$ and $a>0$ such that

$$
\|f\|_{r} \leq \frac{K}{(R-r)^{a}} \quad \text { for all } r \in(0, R) .
$$

Then

$$
\left\|\frac{\partial f}{\partial z_{i}}\right\|_{r} \leq \frac{K e(a+1)}{(R-r)^{a+1}} \quad \text { for all } r \in(0, R) .
$$

Here, $e$ is the base of the natural logarithm.

This type of estimate was first used by Nagumo in his seminal 1942 paper [6] where he considered the classical Cauchy-Kowalevsky Theorem from a new viewpoint. His functional-analytic approach was sufficiently powerful to consider equations that are analytic with respect to the space variable but merely continuous with respect to time. The reader may also refer to Hörmander [3] (see Lemma 5.1.3) for another proof of the lemma.

\section{Proof of Theorem 1.1}

Before we begin with the proof, we point out that the proof of the dual version of Theorem 1.1 is very similar to the one presented in this section. Now, with the dual version on hand, we can simply follow the arguments in [1] (as has been done in [7]) to prove Theorem 1.2. Hence, it is enough to present only the proof of unique solvability.

We will use the method of successive approximations to solve (2). Let $f \in \mathscr{C}^{0}\left([0, T], \mathscr{A}_{R}\right)$ be given, and let $\epsilon>0$ be chosen sufficiently small so that Lemma 2.1 applies and both $\mu(t)$ and $\varphi(t)$ are less than 1 . 
We now define approximate solutions to $(2)$ as follows: let $u^{(0)}=\mathscr{R}[f]$, and for $p \geq 1$,

$$
u^{(p)}=\mathscr{R}\left[B u^{(p-1)}+f\right] .
$$

Here, $\mathscr{R}$ is the inverse operator of $\rho(t) D_{t}+A(t, z, 0)$ and $B$ is the matrix of operators defined by $A(t, z, 0)-A\left(t, z, \mu(t) D_{z}\right)$. Denote by $v^{(p)}$ the difference of the successive approximate solutions, i.e., $v^{(p)}=u^{(p)}-u^{(p-1)}$ for all $p \geq 0$, with the understanding that $u^{(-1)} \equiv 0$.

Note that the convergence of the sequence $\left\{u^{(p)}\right\}$ is equivalent to the convergence of the series $\sum_{p \geq 0} v^{(p)}$. Following the approach in [7], we next observe that $\sum_{p \geq q} v^{(p)}$ may be expressed formally as

$$
\sum_{p \geq q} v^{(p)}=\left(1+(\mathscr{R} B)+\ldots+(\mathscr{R} B)^{d-1}\right) \sum_{p \geq 0} v^{(d p+q)},
$$

where $d$ and $q$ are any two positive integers. Hence, in order to prove that the series $\sum_{p>0} v^{(p)}$ converges, it is sufficient to prove the series $\sum_{p>0} v^{(d p+q)}$ converges for an appropriate choice of integers $d$ and $q$. We will proceed with the following values of the said integers. Set

$$
d=\max \left(n_{i}-n_{j}+1 ; 1 \leq i, j \leq m\right), \quad \gamma=\max \left(n_{i} ; 1 \leq i \leq m\right)
$$

and let $q=d^{2}+2 \gamma$.

Observe further that the difference $A(t, z, 0)-A\left(t, z, \mu(t) D_{z}\right)$ takes the form $\sum_{l=1}^{d} \sum_{|\alpha|=l} A_{\alpha}(t, z)\left(\mu(t) D_{z}\right)^{\alpha}$ for some $A_{\alpha}(t, z)$. Hence we can write $B$ as the sum $\sum_{l=1}^{d} B^{(l)}$, where $B^{(l)}$ is a matrix of differential operators of order $l$ given by $\sum_{|\alpha|=l} A_{\alpha}(t, z)\left(\mu(t) D_{z}\right)^{\alpha}$. (That each operator $B^{(l)}$ is at most of order $d$ is clear from our choice of $d$.) This representation of $B$ allows us to write $v^{(d p+q)}$ as

$$
v^{(d p+q)}=\sum_{1 \leq l_{1}, \ldots, l_{d} \leq d} \mathscr{R} B^{\left(l_{1}\right)} \ldots \mathscr{R} B^{\left(l_{d}\right)} v^{(d(p-1)+q)} .
$$

This is the expression that we will later use in order to estimate the norm of the $i$-th component of the vector $v^{(d p+q)}$.

Lemma 3.1. Let $R_{1} \in(0, R)$. Then there is a constant $C>0$ such that for any $1 \leq i \leq m$, for any $t \in[0, \epsilon]$ and for any $r \in\left(0, R_{1}\right)$, we have

$$
\left\|v_{i}^{(q)}(t)\right\|_{r} \leq C \mu(t)^{d^{2}+n_{i}} \varphi(t)^{\gamma}
$$

Proof. We claim that there is a constant $C_{1}>0$ such that for any $i, j$, $j_{1}, \ldots, j_{q}, k_{1}, \ldots, k_{q}$ and $l_{1}, \ldots, l_{q}$, we have

$$
\left\|\mathscr{R}^{i j_{1}} B_{j_{1} k_{1}}^{\left(l_{1}\right)} \ldots \mathscr{R}^{k_{q-1} j_{q}} B_{j_{q} k_{q}}^{\left(l_{q}\right)} \mathscr{R}^{k_{q} j}\left[f_{j}\right](t)\right\|_{r} \leq C_{1} \mu(t)^{l_{1}+\ldots+l_{q}-\gamma} \varphi(t)^{\gamma}
$$


To show this, we first use the continuity of $f$ on the compact set $[0, \epsilon]$ to bound it above by a constant $K$. We then invoke Lemma 2.2 and (10) to see that $\left\|\mathscr{R}^{k_{q} j}\left[f_{j}\right](t)\right\|_{r}$ is estimated above simply by a constant multiplied by $K$.

Next, we consider the action of the operator $B_{j_{q} k_{q}}^{\left(l_{q}\right)}$, which contains the term $\mu(t)^{l_{q}}$. Since this operator contains a derivative, we have to apply Nagumo's Lemma. Note, however, that we allow $r$ to move only in the interval $\left(0, R_{1}\right)$ and so the derivative becomes a bounded operator. At this point, we have $K \mu(t)^{l_{q}}$ as our bound, where we have denoted again by $K$ the various constants that have arisen. From here, we can now use Corollary 2.3 in estimating the action of $\mathscr{R}^{k_{q-1} j_{q}}$, as well as of all the other $\mathscr{R}$ 's that follow, thanks to the presence of at least one $\mu(t)$. We will apply the corollary with $l=1$ to come up with the estimate $K \mu(t)^{l_{q}-1} \varphi(t)$. (This is possible because each $p(i, j)$ is at least 1.) We repeat the same arguments in estimating the action of the next pairs of $\mathscr{R}$ and $B$ until we have an estimate of the form $K \mu(t)^{l_{q}+\ldots+l_{q-\gamma+1}-\gamma} \varphi(t)^{\gamma}$.

The remaining operators may be estimated similarly, but Corollary 2.3 will have to be applied with $l=0$. After exhausting all the operators, we arrive precisely at the estimate stated in (12). Finally, we only have to recall that $\gamma \geq n_{i}$ and to note that $l_{\nu} \geq 1$ for each $\nu$, so that $l_{1}+\ldots+l_{q}$ is at least $q$.

The next lemma is the main engine in the proof of Theorem 1.1. It gives us a way to estimate recursively the vectors $\left\{v^{(d p+q)}\right\}_{p \geq 0}$.

Lemma 3.2. Let $L$ be a nonnegative integer. If each component $w_{i}$ of the vector $w=\left(w_{1}, \ldots, w_{m}\right)^{\top} \in C^{0}\left([0, \epsilon], \mathscr{A}_{r}\right)$ satisfies

$$
\left\|w_{i}(t)\right\|_{r} \leq(L+1)^{n_{i}} \mu(t)^{d^{2}+n_{i}} \varphi(t)^{\gamma-n_{i}}\left(\frac{\varphi(t)}{R_{1}-r}\right)^{L}
$$

for any $t \in[0, \epsilon]$ and any $r \in\left(0, R_{1}\right)$, then there is a constant $M>0$ independent of $L$ and $w$ such that the estimate

$$
\begin{aligned}
\left\|\mathscr{R}^{i j_{1}} B_{j_{1} k_{1}}^{\left(l_{1}\right)} \ldots \mathscr{R}^{k_{d-1} j_{d}} B_{j_{d} k}^{\left(l_{d}\right)}\left[w_{k}\right](t)\right\|_{r} \leq & M^{l_{1}+\ldots+l_{d}}\left(L+l_{1}+\ldots+l_{d}+1\right)^{n_{i}} \\
& \cdot \mu(t)^{d^{2}+n_{i}} \varphi(t)^{\gamma-n_{i}}\left(\frac{\varphi(t)}{R_{1}-r}\right)^{L+l_{1}+\ldots+l_{d}}
\end{aligned}
$$

holds for any $i\left(=k_{0}\right), j_{1}, \ldots, j_{d}, k_{1}, \ldots, k_{d}(=k)$ and $l_{1}, \ldots, l_{d}$, and also for any $t \in[0, \epsilon]$ and any $r \in\left(0, R_{1}\right)$.

Proof. First, note that in the following, we may assume that $l_{\nu} \leq n_{j_{\nu}}-n_{k_{\nu}}+1$ for all $1 \leq \nu \leq d$, for the assumption on the order of $A_{i j}$ implies that $B_{j k}^{(l)}=0$ whenever $n_{j}-n_{k}+1<l$.

Let us begin by considering the action of $B_{j_{d} k}^{\left(l_{d}\right)}$ on $w_{k}$. This operator has $l_{d}$ derivatives with respect to $z$ and accounts for the presence of $\mu(t)^{l_{d}}$. To estimate 
the derivatives, we apply Nagumo's Lemma $l_{d}$ times to obtain

$$
\left\|B_{j_{d} k}^{\left(l_{d}\right)}\left[w_{k}\right](t)\right\|_{r} \leq K e^{l_{d}}(L+1)_{l_{d}}(L+1)^{n_{k}} \mu(t)^{d^{2}+n_{k}+l_{d}} \frac{\varphi(t)^{L+\gamma-n_{k}}}{\left(R_{1}-r\right)^{L+l_{d}}},
$$

which holds for any $r \in\left(0, R_{1}\right)$. The constant $K$ above is any bound for the norm of the coefficients of the differential operator.

Next, we apply the operator $\mathscr{R}^{k_{d-1} j_{d}}$. By Corollary 2.3, we have the estimate

$$
\begin{aligned}
& \left\|\mathscr{R}^{k_{d-1} j_{d}} B_{j_{d} k}^{\left(l_{d}\right)}\left[w_{k}\right](t)\right\|_{r} \leq C K\left(\frac{1}{c}\right)^{p\left(k_{d-1}, j_{d}\right)-a_{d}} \frac{e^{l_{d}}(L+1)_{l_{d}}(L+1)^{n_{k}}}{\left(R_{1}-r\right)^{L+l_{d}}} \\
& \mu(t)^{d^{2}+n_{k}+l_{d}-a_{d}} \frac{\varphi(t)^{L+\gamma-n_{k}+a_{d}}}{\left(L+\gamma-n_{k}+1\right)_{a_{d}}}
\end{aligned}
$$

for any integer $a_{d}$ between 0 and $p\left(k_{d-1}, j_{d}\right)$ and for any $r \in\left(0, R_{1}\right)$.

We then proceed following the above reasoning. When all the operators acting on $w_{k}$ have been exhausted, we arrive at this estimate:

$$
\begin{aligned}
&\left\|\mathscr{R}^{i j_{1}} B_{j_{1} k_{1}}^{\left(l_{1}\right)} \ldots \mathscr{R}^{k_{d-1} j_{d}} B_{j_{d} k}^{\left(l_{d}\right)}\left[w_{k}\right](t)\right\|_{r} \\
& \leq(C K)^{d} e^{l_{1}+\ldots+l_{d}}\left(\frac{1}{c}\right)^{p\left(i, j_{1}\right)+\ldots+p\left(k_{d-1}, j_{d}\right)-\left(a_{1}+\ldots+a_{d}\right)} \\
& \cdot \frac{(L+1)_{l_{1}+\ldots+l_{d}}(L+1)^{n_{k}}}{\left(L+\gamma-n_{k}+1\right)_{a_{1}+\ldots+a_{d}}\left(R_{1}-r\right)^{L+l_{1}+\ldots+l_{d}}} \\
& \cdot \mu(t)^{d^{2}+n_{k}+l_{1}+\ldots+l_{d}-\left(a_{1}+\ldots+a_{d}\right)} \varphi(t)^{L+\gamma-n_{k}+a_{1}+\ldots+a_{d}} .
\end{aligned}
$$

Now each $l_{\nu}$ is at most $n_{j_{\nu}}-n_{k_{\nu}}+1$, so that $l_{\nu}+n_{k_{\nu}}-n_{k_{\nu-1}} \leq p\left(k_{\nu-1}, j_{\nu}\right)$. (Recall that $k_{0}=i$ and $k_{d}=k$.) Summing up over all $\nu$ 's gives $l_{1}+\ldots+l_{d}+n_{k} \leq$ $\sum_{\nu=1}^{d} p\left(k_{\nu-1}, j_{\nu}\right)+n_{i}$. This tells us that it is possible to choose $a_{\nu}$ 's in such a way that $l_{1}+\ldots+l_{d}+n_{k}=a_{1}+\ldots+a_{d}+n_{i}$. With this choice of $a_{\nu}$ 's , we can further simplify (13) to obtain the desired estimate.

We are now ready to prove the convergence of $\sum_{p=0}^{\infty} v^{(d p+q)}$. We start with the estimate in Lemma 3.1, to which we repeatedly apply Lemma 3.2. After $p$ successive applications of the latter lemma, we obtain for $t \in[0, \epsilon]$ the estimate

$$
\left\|v_{i}^{(d p+q)}(t)\right\|_{r} \leq \sum_{1 \leq l_{1}, \ldots, l_{d p} \leq d} C M^{l}(l+1)^{n_{i}} \mu(t)^{d^{2}+n_{1}} \varphi(t)^{\gamma-n_{i}}\left(\frac{\varphi(t)}{R_{1}-r}\right)^{l}
$$

where for brevity we have denoted by $l$ the sum $l_{1}+l_{2}+\ldots+l_{d p}$. Note that this sum is at least $d p$ and at most $d^{2} p$. Since $\left(\begin{array}{c}p-1 \\ d-1\end{array}\right)$, the number of ways one can form the sum $p$ from $d$ not necessarily distinct integers each of which is at 
least 1 , is not more than $2^{p}$, we can further estimate as follows:

$$
\begin{aligned}
\left\|v_{i}^{(d p+q)}(t)\right\|_{r} & \leq C \mu(t)^{d^{2}+n_{i}} \varphi(t)^{\gamma-n_{i}} \sum_{d p \leq l \leq d^{2} p}(l+1)^{n_{i}}\left(\frac{2 M \varphi(t)}{R_{1}-r}\right)^{l} \\
& \leq C \mu(t)^{d^{2}+n_{i}} \varphi(t)^{\gamma-n_{i}}\left(\frac{2 M \varphi(t)}{R_{1}-r}\right)^{d p} p\left(d^{2}-d\right)\left(d^{2} p+1\right)^{n_{i}},
\end{aligned}
$$

if we fix $r$ and choose $\epsilon$ small enough so that for $|t|<\epsilon$ we have $2 M \varphi(t)<R_{1}-r$. Having established this, it is now easily seen that the series $\sum_{p=0}^{\infty} v^{(d p+q)}$ is indeed convergent in $C^{0}\left([0, \epsilon], \mathscr{A}_{r}\right)$. This implies that the approximate solutions $u^{(p)}$ converge to a solution $u$ in $\mathscr{C}^{0}\left([0, \epsilon], \mathscr{A}_{r}\right) \cap \mathscr{C}^{1}\left((0, \epsilon], \mathscr{A}_{r}\right)$. This proves the existence part of Theorem 1.1. The uniqueness of the solution can be proved in a similar manner and so we may omit the details.

Acknowledgment. This paper was completed during the stay of the first author at the Institute of Mathematics and Scientific Computing of the University of Graz; his stay in Graz was under the auspices of the Austrian Exchange Service. He would like to thank the members of the Institute and the staff of the ÖAD for their hospitality and support. All three authors were supported by the National Science and Research Institute (Project Code: MAT-03-2-02) of the University of the Philippines - Diliman.

\section{References}

[1] Baouendi, M. S. and C. Goulaouic: Cauchy problems with characteristic initial hypersurface. Comm. Pure Appl. Math. 26 (1973), 455 - 475.

[2] Elschner, J.: Einige Bemerkungen zu einer Arbeit von Baouendi und Goulaouic. Beiträge Anal. 12 (1978), 185 - 198.

[3] Hörmander, L.: Linear Partial Differential Operators. Berlin: Springer 1963.

[4] Koike, M.: Volevič systems of singular nonlinear partial differential equations. Nonlinear Anal. 24 (1995), 997 - 1009.

[5] Lope, J. E. C.: Existence and uniqueness theorems for a class of linear Fuchsian partial differential equations. J. Math. Sci. Univ. Tokyo 6 (1999), 527 538.

[6] Nagumo, M.: Über das Anfangswertproblem partieller Differentialgleichungen. Jap. J. Math. 18 (1942), $41-47$.

[7] Tahara, H.: On a Volevič system of singular partial differential equations. J. Math. Soc. Japan 34 (1982), 279 - 288.

[8] Tahara, H.: On the uniqueness theorem for nonlinear singular partial differential equations. J. Math. Sci. Univ. Tokyo 5 (1998), 477 - 506.

Received 28.06.2004 